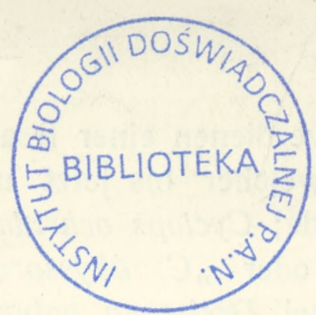

\title{
Uber die Eigentümlichkeiten des Zooplanktons des Ohridsees.
}

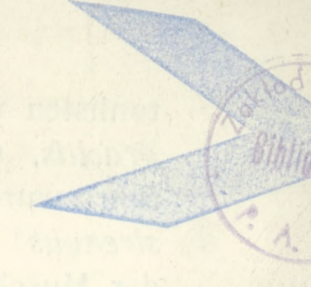

Von

Z. Koź min ski, Wigry.

Dank der Liebenswürdigkeit des Herrn Professor Dr. S. Stanković habe ich eine reichhaltige Planktonsammlung aus dem Ohridsee zur Bearbeitung erhalten. Dieses Material, welches aus etwa 200 Proben besteht, wurde mittels zwei Modellen der quantitativen Planktonnetze (Nr.Nr. 12 und 20 der Müller'schen Seidengaze) ausschliesslich in der pelagischen Region des Sees im Jahre 1926/27 (3. VIII. 26 -- 24. VII. 27) und im Sommer $192 \mathrm{~J}$ (19. V - 18. IX) in beinahe monatlichen Zeitabständen gesammelt und unterliegt jetzt einer eingehenden quantitativen Bearbeitung. In der vorliegenden Mitteilung möchte ich kurz das Zooplankton der pelagischen Region des Ohridsees qualitativ charakterisieren, soweit dies beim jetzigen Stand der Untersuchung der Planktonproben möglich ist. Die Fragen über die Gesamtproduktion des Zooplanktons sowie über die vertikale Verbreitung einzelner Arten im See werden einer künftigen quantitativen Arbeit vorbehalten.

Das Zooplankton des Ohridsees wurde schon von einigen Autoren (R i chard 1892, Steuer 1900, Ge orgevitch 1905, 1907, P arenzan, 1930, 1931, Brian 1930, Forti 1930, Chappuis bei Stanković 1931, Kiefer 1932) untersucht und seine qualitative Zusammensetzung wurde von ihnen beinahe vollkommın bekanntgegeben. In der umstehenden Tabelle sind die Arten angeführt, welche in dem von mir untersuchten Material festgestellt wurden'). Im Vergleich mit den früher veröffentlichten Plank-

1) Bei der Bestimmung der Arten einzelner Gruppen waren mir in liebenswürdiger Weise behilflich: Herr St. Feliks i a k, Warszawa, - Lamellibranchiata und Herr Dr. J. W i s z n i e w s k i, Wigry, - Rotatoria. Ich spreche den genannten Herren für Ihre Bereitwilligkeit meinen herzlichen Dank aus. Ich bin auch Frl. M. Wi e r zbicka, Wilno, welche mit mir die Mühe der quantitativen Bearbeitung des Ohridmaterials unternehmen wollte, zum besten Dank verpflichtet. 
tonlisten verdienen einer Beachtung folgende Arten: Diaptomus gracilis, welcher bis jetzt meistens als "Diaptomus sp." angeführt wurde; Cyclops ochridanus, welcher unter dem Namen „C. strenuus" oder "C. abyssorum" angegeben wurde; die Larven der Muschel Dreissena polymorpha sowie endlich einige Rotatorienarten, welche für die Ohridfauna neu sind oder $z$. T. vorher durch andere Speziesnamen bezeichnet wurden. In die Zusammensetzung des Ohridzooplanktons können ausserdem vielleicht noch Daphnia longispina O. F. M. (vgl. P a re n zan 1930) und einige Rotatorienarten hineingehen, namentlich Polyarthra (trigla Ehr.), die von Forti (1930), S t a n k ović (1931) und P a r e n$\mathrm{z}$ an (1931) angegeben wurde, deren Anwesenheit in dem von mir untersuchten Material jedoch nicht festgestellt werden konnte; auf den Gesamtcharakter des Ohridplanktons sollen aber diese Arten keinen grösseren Einfluss ausüben.

\section{Die pelagische Planktonfauna des Ohridsees}

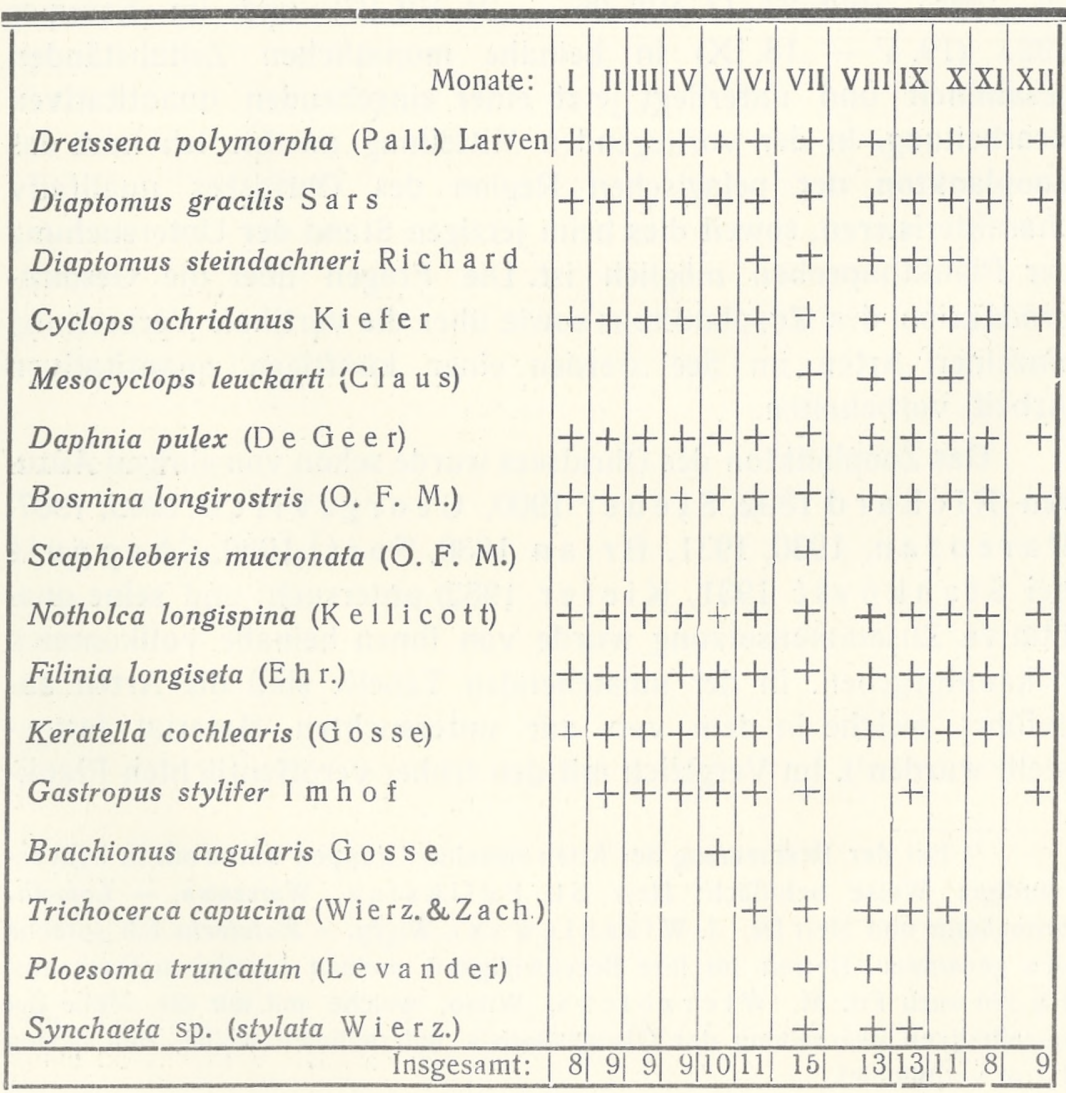


Wie aus der beigefügten Tabelle folgt, ist die qualitative Zusammensetzung des besprochenen Planktons ziemlich artenarm und eintönig: man könnte wohl mit Recht erwarten, daß das Zooplankton eines so grossen oligotrophen Sees, dessen Reichtum an vielen oft sehr eigentümlichen Tierarten weit berühmt ist, eine mehr mannigfaltige Entwicklung zeigen wird. Trotz seiner relativen Artenarmut zeichnet sich aber dieses Plankton durch einige interessante Eigentümlichkeiten aus, welche hier näher besprochen werden.

Ein jeder Planktologe, der mit der pelagischen Fauna der mitteleuropäischen Seen einigermassen vertraut ist, wird gewiss durch die Tatsache erstaunt, daß im Plankton der pelagischen Region des Ohridsees, neben den Cladoceren, Copepoden und Rotatorien, welche bekanntlich den Hauptbestandteil des Seenplanktons bilden, eine quantitativ hervorragende Rolle die Mollusken, und zwar die Muschellarven, spielen. Das massenhafte Auftreten dieser Larven im Plankton der grossen Seen der Balkanhalbinsel wurde bereits von früheren Autoren erwähnt (R ichard 1892, Georgevitch 1905, Forti 1930, Parenzan 1931), die genaue systematische Stellung dieser Larven blieb jedoch ungeklärt ${ }^{2}$ ). Wie bekannt, wurden bis jetzt im Süßwasserplankton die Larven nur einer Muschelart, und zwar von Dreissena polymorpha, nachgewiesen. Da die Dreieckmuschel gerade im Ohridsee nach Stanković (1931, S. 563) sehr häufig ist, schien es sofort sehr wahrscheinlich, daß die hier beobachteten Larven eben der genannten Art angehören. Diese Vermutung wurde durch eine Feststellung von Herr St. F elik s i a k (Poln. Zoologisches Staatsmuseum, Warszawa) vollkommen bestätigt; es wurde nämlich im Scheitelteil der Schale einiger sehr junger, nur $1 \mathrm{~mm}$-langer, aber schon festsitzender Dreissena-Exemplare aus dem Ohridsee, die sich in den Sammlungen des Zoologischen Staatsmuseums in Warszawa gefunden haben, die Anwesenheit einer Larvalschale (der sog. Prodissoconcha) beobachtet, und zwar von einem Aussehen, das demjenigen der Schalen der planktischen Muschellarven vollkommen ähnelt. Somit scheint die systematische Zugehörigkeit dieser Larven ziemlich sicher aufgeklärt zu sein; die künftige eingehende Bearbeitung der Ohridlamellibranchiaten, die bisnun leider fehlt, wird aller Wahrscheinlichkeit nach diese Bestimmung bestätigen.

2) Sie wurden von Richard (1892) und Ge orgevitch (1905) als „Cycladida“, von P a r e n z a n (1931, S. 36) dagegen als „Unio?“ bestimmt. 
In vielen Seen Mitteleuropas, welche zahlreiche Kolonien der Dreieckmuschel beherbergen, treten ihre Larven im Plankton der pelagischen Region - soweit mir aus den spärlichen diesbezüglichen Literaturangaben sowie aus eigener Erfahrung bekannt ist - nur sporadisch, wenn auch mitunter ziemlich zahlreich auf; diese freischwimmenden Larven dürfen hier nicht dauernd verweilen, da ihr planktisches Leben, soweit bekannt ist, auf einen ganz kurzen Zeitraum beschränkt ist. Im Gegensatz zu diesen VerhäItnissen treten die Muschellarven im Plankton des Ohridsees während des ganzen Jahres, sowohl nahe der Wasseroberfläche, wie auch in den tieferen Wasserschichten, in ungeheuren Mengen auf, indem sie mitunter, namentlich im Winter, eine. quantitativ dominierende Form bilden. Es verdient auch hervorgehoben zu werden, daß im Sommer und Herbst (Juli - Dezember). neben den größeren Larven auch ganz kleine Exemplare sehr zahlreich vorkommen, während in den übrigen Jahreszeiten nur die Larven von ungefähr gleicher Größe angetroffen werden. - Der Entwicklungszyklus und die Biologie der Dreissena polymorpha im Ohridsee scheinen demnach etwas verschieden zu sein, als in den mitteleuropäischen Seen und sie verdienen zweifelsohne eines eingehenden Spezialstudiums.

Die Rotatorien, welche im Plankton des Ohridsees festgestellt wurden (s. Tabelle), repräsentieren einen wohl kosmopolitischen Komplex der in den Süßwasserseen gewöhnlich vorkommenden Arten. Unter den Crustaceen finden wir dagegen manche interessante Formen. Es sind nämlich vor allem die Diaptomiden, welche dank ihrer geographischen Differenzierung besonders bemerkenswert sind. Im Ohridsee finden wir zwei Arten dieser Familie vor.

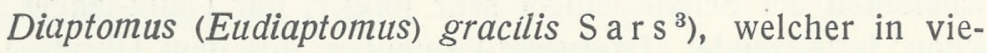
len Ländern Eurasiens, namentlich im Norden, sehr verbreitet: ist, wurde im Plankton der südeuropäischen Seen ziemlich selten angetroffen, indem er dort meistens durch die Formen der vul-

3) Das Vorkommen eben dieser Art im Ohridsee halte ich für vollkommen sicher; die untersuchten Exemplare zeichnen sich durch alle für $D$. gracilis charakteristische Eigenschaften aus. Eine gewisse, wenn auch meines Erachtens geringfügige Differenz gegenüber den mir aus den Seen Polens bekannten Formen stellt die etwas kürzere erste Antenne dar, welche zurückgeschlagen kaum das Körperende des Weibchens erreicht; dem drittletzten Gliede der männlichen Greifantenne fehlt bei den Ohridexemplaren der charakteristische Fortsatz, dieses Glied ist dagegen in eine kleine hyaline Lamelle ausgerüstet. Diese Unterschiede berechtigen aber nicht, wie mir scheint, die Ohridform als eine besondere Varietät aufzufassen. 
garis- oder graciloides-Gruppe ersetzt wird. D. gracilis stellt demnach ein ziemlich eigentümliches Merkmal des Ohridplanktons dar; er kommt in diesem See in grosser Menge während des ganzen Jahres vor, ihre maximale Entwicklung erreicht diese Art jedoch im Winter (Dezember-März). Im Sommer (VII-IX) kann man nur die Weibchen beobachten. Es ist eine interessante Tatsache, daß in den Monaten von Februar bis Juni einschliesslich treten nebeneinander die Weibchen von zwei stark divergierenden Körpergrössen auf, wie es scheint - ohne irgendwelcher Übergänge: die kleineren Weibchen überschreiten nicht die Totallänge von etwa $1.2 \mathrm{~mm}$, während die grösseren eine solche von ungefähr $1.5 \mathrm{~mm}$ erreichen; in den übrigen Monaten wurden nur die grösseren Weibchen angetroffen. Ich verweise hier auf eine interessante Arbeit von G u r n e y (1929), welcher diese Erscheinung, auch bezüglich $D$. gracilis, ziemlich ausführlich beschrieben und diskutiert hat.

Die zweite Diaptomidenart des Ohridsees, Diaptomus (Arctodiaptomus) steindachneri $\mathrm{R}$ i c h a r d, bildet, wie es scheint, eine endemische, nur den mazedonischen Seen eigene Form. Krmpotić (1925) hat zwar eine Reihe von Standorten dieser Art aus den Srijemer Sümpfe in Nordjugoslavien angegeben; nach der Beschreibung dieses Verfassers urteilend, könnte man aber schliessen, daß es sich eigentlich nicht um D. steindachneri, sondern wahrscheinlich um eine nahe verwandte, im Jahre 1925 noch nicht beschriebene Art, und zwar um D. dentifer S mirnov (1928), handelte. Dasselbe gilt wohl auch für die von M o n t i (1934) angegebenen albanischen Fundorte von "D. steindachneri". Die gegenseitigen Verwandschaftsbeziehungen dieser zwei Formen verlangen übrigens unbedingt einer näheren Untersuchung. - D. steindachneri kommt im Ohridsee nur in den Sommermonaten (VI-X) vor und bildet zu dieser Zeit einen quantitativ wichtigen Bestandteil des Zooplanktons der pelagischen Region des Sees.

Die Familie Cyclopidae ist in der pelagischen Zone des Ohridsees auch durch zwei Arten vertreten. Mesocyclops leuckarti (C la us) bildet wohl eine kosmopolitische und wahrscheinlich ziemlich eurytope Form. In dem von mir untersuchten Material kommt er in einer sehr beschränkten Individuenanzahl nur in den Sommerproben (VII-X) vor. In manchen Jahren dürfte er indessen in grossen Mengen auftreten, wie dies die Angabe von B r i a n (1930) beweist.

Der zweite pelagische Repräsentant der Familie Cyclopidae, Cyclops ochridanus Ki fer, ist für das Plankton des Ohridsees 
sehr charakteristisch und kommt während des ganzen Jahres in grosser Menge vor. Diese unlängst von K i ef e r (1932) beschriebene, sehr eigentümliche und in mancher Hinsicht recht primitive Art, gehört der Gruppe strenutus, deren morphologische und ökologische Differenzierung in den letzten Jahren den Gegenstand eingehender Untersuchungen bildete (vgl. Ko źmiński 1933). Eine genaue morphometrische Charakteristik von $C$. ochridanus sowie seine Verwandschaftsbeziehungen $\mathrm{zu}$ anderen Arten der Untergattung Cyclops beabsichtige ich an anderer Stelle zu veröffentlichen. - Diese Art ist bis jetzt nur aus dem Ohridsee bekannt, man könnte jedoch erwarten, daß sie sich vielleicht auch in manchen anderen Seen Mazedoniens finden lässt, aus welchen nicht einmal "Cyclops strenuts" angegeben wurde. Jedenfalls scheint es sehr wahrscheinlich, daß C. ochridanus, ähnlich wie Diaptomus steindachneri, eine endemische, nur den grossen mazedonischen Seen (oder sogar vielleicht nur dem Ohridsee?) eigene Form darstellt.

Die strenuus-Fauna der südeuropäischen Seen scheint im allgemeinen viel mannigfaltiger und interessanter zu sein, als man es aus der älteren Literatur schliessen konnte. In dem Vranasee auf der Insel Cherso (Adria) lebt pelagisch Cycl. strenuus subsp. vranae $\mathrm{Koźm}$.; nahe verwandte Formen scheinen in manchen Seen Italiens vorzukommen (v.gl. Los it o 1904, B aldi 1924, Stella 1934). Als einziger Repräsentant der strenuus-Gruppe im Ohridsee tritt $C$. ochridanus auf; in dem nahe gelegenen Prespasee konnte ich dagegen die. Anwesenheit von Cyclops vicinus Uljanin - einer sehr weit verbreiteten Art - feststellen; in der mir zur Verfügung stehenden Planktonprobe aus diesem See war dagegen kein einziges Exemplar von $C$. ochridanus vorhanden. Man kann also nicht ausschliessen, daß „Cyclops strenuus" aus den mazedonischen Seen eben $C$. vicinus ist, mit einer Ausnahme des Ohridsees, in welchem er durch $C$. ochridanus ersetzt ist. Die Ursachen dieser Differenzen können z. T. historischer Natur sein, es erscheint aber wahrscheinlicher, daß es sich vielmehr um ökologische Unterschiede der einzelnen Seen handelt.

Unter den pelagischen Cladoceren des Ohridsees verdient nur das Vorkommen von Daphnia pulex (De Geer) einer näheren Besprechung. Bekanntlich treten die Repräsentanten dieses Formenkreises im Plankton der eigentlichen Seen in Europa ziemlich selten auf; soweit mir bekannt, sind es vor allem die Gebirgsseen der Alpen und der Hohen Tatra, in welchen die pulex-Formen pelagisch vorkommen. In den nordamerikanischen 
Seen dagegen erreichen diese Formen, wie das auch aus den neuen Untersuchungsergebnissen von W olt e reck (1932) folgt, eine grosse Entwicklung und Differenzierung. Die pulex.Kolonie des Ohridsees, die eine bedeutende Variabilität aufweist, ihr Lebenszyklus und vertikale Verbreitung in dem See werden den Gegenstand einer besonderen Untersuchung bilden, die bereits unternommen wurde. - Die übrigen Cladocerenarten sind sehr weit verbreitet; Bosmina longirostris (O. F. M.) tritt im Plankton des Ohridsees während des ganzen Jahres auf, sie ist aber meistens wenig zahlreich. Scapholeberis mucronata (O. F. M.) wurde dagegen nur im Sommer in wenigen Exemplaren angetroffen. Was endlich das Auftreten der Art Daphnia longispina O. F. M. anbetrifft, welche von P arenzan (1930) für Ohrid angegeben worden ist, so muss diese Frage unentschieden bleiben, bevor ein näheres Studium der Daphnia-Kolonien dieses Sees nicht durchgeführt wird. Ich habe zwar beim Durchmustern zahlreicher Daphnien meines Materials neben solchen Exemplaren, die einen Nebenkamm an der Endkralle besassen, auch solche angetroffen, die keinen deutlichen Nebenkamm aufzuweisen schienen; doch dürften die letzteren wahrscheinlich auch der Art D. pulex zugerechnet werden. Es ist möglich, daß eben ähnliche Exemplare von $\mathrm{P}$ a re $\mathrm{nz}$ a n als $D$. longispina anerkannt wurden; ob diese Behauptung berechtigt sei, werden wohl spätere eingehende Untersuchungen beweisen.

Damit sei die Besprechung einzelner Plankter des Ohridsees abgeschlossen. Was den allgemeinen Charakter dieses Planktons anbetrifft, so möchte ich noch die Aufmerksamkeit auf eine sehr interessante ökologische Frage lenken. - Es ist eine bekannte Tatsache, daß manche Arten, welche in Mitteleuropa hauptsächlich in grösseren und tieferen Seen eupelagisch leben (z. B. Bythotrephes longimanus, Diaptomus graciloides, Cyclops scutifer etc.), weiter im Norden auch ganz kleine und seichte Wasserbecken bewohnen. Es drängt sich jetzt die Frage auf, ob ein ähnliches Verhältnis sich zwischen der mit t e leuropäischen Kleingewässer- und Seeuferfauna einerseits und dem Plankton der pelagischen Zone der südeuropäischen S e en andererseits nicht nachweisen lässt? - Das Plankton des Ohridsees weist in der Tat einige bemerkenswerte Zeichen auf, welche eine gewissermassen positive Antwort auf diese Frage zu geben gestatten. So lebt z. B. im Ohridsee pelagisch Daphnia pulex, die in Mitteleuropa zu den typischen Bewohnern der kleinen stark verunreinigten Gewässer und gleichzeitig der Litoralfauna einiger Seen gehört. 
Dasselbe Verhalten scheint unter den Cladoceren auch die im Ohridsee pelagisch auftretende Scapholeberis mucronata zu verraten. Weiterhin weist der eulimnetische Diaptomus steindachneri eine bemerkenswerte weitgehende Verwandschaftsbeziehung zu $D$. dentifer Smirnov, welcher nach Rylov (1930) in den Kleingewässern Zentralrusslands auftritt. Endlich zeigt auch Cyclops ochridanus einige interessante morphologische Ähnlichkeitsbeziehungen zu $C$. furcifer $\mathrm{Claus,} \mathrm{einer} \mathrm{echten} \mathrm{Kleingewässerform}$ Mittel- und Nordeuropas. Bei der allgemeinen Artenarmut der Planktonfauna des Ohridsees erteilen eben die erwähnten Formen dem Gesamtplankton dieses Sees ein besonderes Gepräge, um so mehr, als es sich hier meistens um zahlreich auftretende und wohl merkwürdigste Planktonkomponenten handelt.

Ausser den oben berührten positiven Eigenschaften verdienen auch einer kurzen Besprechung einige negative Merkmale des Ohridplanktons. Es wurde schon oben auf die relative Artenarmut dieses Planktons hingewiesen, welche ziemlich unerwartet ist, wenn man den oligotrophen Charakter des Sees und den Reichtum seiner Uferfauna in Betracht zieht. Diese Artenarmut drückt sich nicht nur im Fehlen vieler nordischer oder weit verbreiteter Planktonformen Europas aus, sondern auch - was besonders auffallend ist - im Fehlen mancher Formen, welche eben in den Seen der Balkanhalbinsel gemein sind. Ich führe nur solche gewöhnliche Arten an, wie Diaphanosoma brachyurum (L i é v i n), Leptodora kindtii ( $\mathrm{F} \mathrm{o} \mathrm{k} \mathrm{e}$ ), Diaptomidenarten aus der Gruppe vulgarts oder graciloides; unter den Rotatorien bemerkenswert ist das Fehlen eines solchen Ubiquisten, wie Keratella quadrata (M ü $11 \mathrm{e} \mathrm{r).} \mathrm{Man}$ findet auch im Plankton des Ohridsees keine Arten, welche als ausgesprochen mediterran oder pontisch bezeichnet werden könnten. Ähnlich, wie die grossen Reliktenseen, Baikal und Tanganyika, zeichnet sich somit auch der Ohridsee durch eine ziemlich eigentümliche, aber qualitativ wohl kümmerliche, sozusagen def e kte $\mathrm{Zus}$ a m m e n s et zung des pelagischen $\mathrm{Z}$ o o plankt on s aus. Diese merkwürdige Erscheinung verdient zweifellos einer besonderen Beachtung.

Eine negative Eigenschaft des Ohridplanktons bilden auch seine ziemlich schwach ausgeprägte Saisonunterschiede. Wie aus der beigefügten Tabelle, wo das Auftreten einzelner Formen in einzelnen Monaten vermerkt ist, ersichtlich, könnte die Planktonzusammensetzung in dieser Hinsicht in folgende Gruppen eingeteilt werden: 1) 9 perennierende Formen; 2) 5 Sommerformen und 3) einige Arten, deren Verhältnis zur Jahreszeit nicht ganz klar her- 
vortritt. Die ausgesprochene Artenarmut des Winterplanktons und das vollkommene Fehlen echter Winterformen sind wohl auffallend; nur zwei Formen (Dreissena-Larven und Diaptomus gracilis) treten im Winter in größerer Individuenzahl, als im Sommer auf. Die Mehrheit (9 auf 16) der Zooplanktonarten kommt während des ganzen Jahres hindurch vor und vermehrt sich wahrscheinlich azyklisch.

Diese Übersicht der Eigenschaften des Ohridzooplanktons belehrt, daß dieses Plankton trotz gewisser Eintönigkeit doch manche spezifische Zeichen aufweist; das Auftreten zweier wahrscheinlich endemischer Planktonarten in einem Süßwassersee stellt jedenfalls schon für sich selbst eine ziemlich eigentümliche Erscheinung dar. Obwohl gewisse Versuche einer Aufklärung der Herkunft der balkanischen Planktoncrustaceen seinerzeit unternommen worden waren (Ge orgevitch 1905, 1907, vgl. auch das Referat von B r e hm, Intern. Rev. Hydr. 1908), doch scheint es mir, daß es auch heute noch zu schwer sein würde eine befriedigende $\mathrm{k}$ a u s a $1 \mathrm{e}$ Interpretation der qualitativen Zusammensetzung des Ohridzooplanktons sowie mancher, dieses Plankton betreffender, biologischer Erscheinungen zu geben. Es erscheint z. B. zur Zeit leider unmöglich diese Verhältnisse an die ausserordentlich wichtigen und interessanten zoogeographischen Erwägungen, die unlängst von S t a n k ovi ć (1931) publiziert wurden, anzuknüpfen.

Es sei mir gestattet auch hier Herrn Professor Dr. S. S t a nković für seine ganz ausserordentliche Bereitwilligkeit und Liebenswürdigkeit, dank welchen ich imstande war das Zooplankton eines der interessantesten Seen Europas zu untersuchen, meinen verbindlichsten Dank auszusprechen.

Hydrobiologische Station am Wigrysee, Polen.

\section{Literaturverzeichnis.}

B a 1 d i, F. 1924. I copepodi lariani. La limnologia del Lario, Rina Monti. Roma. B ria n, A. 1930. Copepodi dei laghi albanesi di Ochrida e di Malik. Atti Accad. Veneto-Trentino-Istriana. 21. Citta di Castello.

Forti, A. 1930. Osservazioni biologiche sopra alcuni laghi dell'Albania orientale. Atti Ac. Sc. Ven.-Trent.-Istr. 21. Selci Umbro.

G e orgevitch, Ž. 1905. Die Planktonorganismen der großen Seen der Balkanhalbinsel. Glas. d. serb. Akad. Wiss. Beograd.

1907. Les organismes planctoniques des grands lacs de la péninsule Balkanique. Mem. Soc. Zool. de France. 20. Paris.

- $\quad$ 1907. Ein Beitrag zur Kenntnis der Diaptomiden Serbiens. Zool, Anz. 1907.

G u r n e y, R. 1929. Dimorphism and rate of growth in Copepoda. Intern. Rev. Hydr. 21. Leipzig. 
K o ź m i ń s k i, Z. 1933. Badania morfometryczne i ekologiczne nad oczlikami (Cyclopidae) z grupy strenuus. Arch. Hydrob. i Ryb. 7. Suwalki.

K r m p otić, I. 1925. Beitrag zur Kenntnis der Entomostraken und Rotatorien, insbesonders der Diaptomiden Kroatiens und Slavoniens. Arch. f. Hydr. 15. Stuttgart.

L o sito, C. 1904. Entomostraci pelagici del lago di Bracciano. Lavori eseguiti d. staz. piscicolt. Roma.

M o nti, R. 1934. Contríbuto all'idrobiologia delle alpi Albanesi. Atti Soc. Ital. Sc. Nat. 73. Milano.

Parenzan, P. 1930. Nota sui Cladoceri dei grandi laghi dell'Albania orientale. Atti Accad. Sc. Ven.-Trent.-Istr. 21. Selci Umbro.

P a r e n $\mathrm{z}$ a n, P. 1931. Cladoceri d'Albania con brevi notizie morfologiche ed idrologiche sui grandi laghi albanesi. Atti Accad. Sc. Ven.-Trent.-Istr. 22. Selci Umbro.

R i c h a r d, J. 1892. Animaux inférieurs, notamment Entomostracés, recueillis par M. Steindachner dans les lacs de la Macédoine. Ann. Hofmus. Wien.

Rylov, W. M. 1930. Fresh-water Calanids of U.S.S.R. in Keys to determ. of Fresh-water organisms of the U.S.S.R.A. I. Leningrad.

S m i r no v, S. S. 1928. Über eine neue Diaptomus-Art (Copepoda) aus Zentralrussland. Zool. Anz. 78. Leipzig.

Stanković, S. 1931. Die Fauna des Ohridsees und ihre Herkunft. Arch. f. Hydrob. 23. Stuttgart.

Ste 11 a, E. 1934. I planctonti del lago di Resia durante l'estate subacquea. R. Istit. Lombardo Sc. Lett. Rendiconti. 67. Milano.

St e u e r, A. 1900. Die Diaptomiden des Balkan, zugleich ein Beitrag zur Kenntnis des Diapt. vulgaris Schmeil. Sitz.-ber. Kaiserl. Ak. Wiss. Wien. Math. Naturwiss. C1. Bd. CIX. Abt. 1.

W o 1 te reck, B. 1932. Races, associations and stratification of pelagic Daphnids in some lakes of Wisconsin and other regions of U. S. A. and Canada. Trans. Wisc. Acad. Sc. 27. 1932.

\section{D is kus sion}

H. K a $1 \mathrm{~m}$ us, Prag: Da die Grössenverteilung der DreissensiaLarven über das Jahr nicht wesentlich schwankt könnte man vielleicht annehmen, daß die D.-Larven, die ja in einem Süßwassersee zweifellos nicht optimale Existenzbedingungen finden, dort eine sehr verlängerte Larvenperiode durchmachen. Diese Arbeitshypothese scheint deshalb naheliegend, weil, wie $\mathrm{Kalmus}$ und Hagmeier auf Helgoland feststellten, Molluskenlarven (bez. Austernlarven) unter leicht abgeänderten, d. h. verschlechterten Bedingungen (Kultur) z.war sehr resistent sind aber die Ansatzgrösse monatelang nicht erreichen. Durch nichtoptimale Bedingungen verursachte extreme Verlängerung der Larvalzeit könnte also das auffallende Vorkommen der Dreissensia-Larven im Ohridplankton erklären. 